\title{
Numerical Analysis of Isotropy Electromagnetic Sensor Measurement Error
}

\author{
R. Hart'anský, V. Smieško, L. Maršálka \\ Institute of Electrical Engineering, Faculty of Electrical Engineering and Information Technology, Slovak University of \\ Technology in Bratislava, Slovakia, e-mail: rene.hartansky@stuba.sk
}

The article deals with classification and quantification of electromagnetic field measurement errors in case an isotropic sensor as a field probe is used. The focus is mainly on the error of measurement method, resulting from mutual interaction of the field probe sensors associated with the origin of the so-called mutual impedance.

Keywords: Electromagnetic field, sensors, measurement error.

\section{INTRODUCTION}

$\mathrm{A}$ SUFFICIENT ACCURACY of measuring the amplitude of the electromagnetic (EM) field is required in many branches of electrical engineering (e.g., during EM compatibility testing of electrical devices). The measurement is performed in many cases in a far field. Then it is sufficient to measure only one component of the EM field while the other can be easily estimated. Despite this simplification, measured component of the EM field is a vector. This means that we need to know all the vector components $(\mathrm{x}, \mathrm{y}, \mathrm{z})$, which determine the final amplitude of the measured vector. To determine the mentioned components of the vector an omnidirectional probe of the EM field must be used. In general, such a probe is made up of three orthogonal sensors, which are oriented in mentioned vector components, the output voltage of the sensors is determined as follows:

$$
U_{E}=\sqrt{U_{x}^{2}+U_{y}^{2}+U_{z}^{2}}
$$

In order to ensure sufficient accuracy, the sensors have to meet a series of conditions:

- The frequency-independence [1];

- Sufficient output voltage [1];

- Sensitivity only to one component of the electric field vector [1], [5];

- Linearity [2];

- No (negligible) interaction with surrounding objects.

In the scientific literature, attention is given to the first four conditions; however, the fifth condition is not addressed. Therefore, this article deals with the mutual interaction of sensors, and their effects on the error of the EM field measurement.

\section{MUTUAL IMPEDANCE}

If a conductive object is situated in the EM field, there is an interaction between the field and the object. This interaction is reflected in changing the distribution of the EM field geometry and creation of an electric current or electromotive voltage on this conductive object. If there are other objects in the EM field, also other interactions arise; not only interactions between field and object, but also interaction between the objects. This interaction is known as mutual impedance in the antenna theory. The creation of mutual impedance reflects in changing of voltage and current conditions in an EM field of the objects, similarly to the theory of linear circuits. The mutual impedance may be calculated by several methods. All the methods are based on the interaction between the incident and radiated EM field of the studied objects (for example: two wires with lengths $2 \mathrm{~h}$ ). According to [3] or [4], the mutual impedance may be expressed as:

$$
Z_{21}=-\frac{1}{I_{1 i} I_{2 i}} \int_{-h}^{h} I_{2}\left(z^{\prime}\right) E_{z 21}\left(z^{\prime}\right) d z^{\prime}
$$

where $I_{2}\left(z^{\prime}\right)$ is a current distribution at wire $2, E_{z 21}\left(z^{\prime}\right)$ is E-field component radiated by wire 1 , which is parallel to wire $2 ; \mathrm{I}_{1}, \mathrm{I}_{2 \mathrm{i}}$ are wires' input currents. As it was mentioned above, the mutual impedance affects the induced voltage at the input of wire 1 and wire 2 as follows:

$$
U_{1}=U_{11}+Z_{21} I_{2}
$$

where $U_{1}$ is driving point voltage of wire 1 , if wire 2 is present. $\mathrm{U}_{11}$ is driving point voltage of wire 1 without wire 2.

Let us return to the EM field probe, which consists of three sensors (three wires). If one of the sensors captures only the $\mathrm{X}$-axis component of the E-field, its output voltage will be:

$$
U_{x}=k_{x} E_{x}
$$

where $k_{x}$ is the coefficient including form and property of the $\mathrm{x}$-sensor.

If two sensors for the x-axis and y-axis component of the EM field are situated close to each other, then the output voltage of them will be [4]:

$$
\begin{aligned}
& U_{x 1}=U_{x}+Z_{x y} I_{y} \\
& U_{y 1}=U_{y}+Z_{y x} I_{x}
\end{aligned}
$$


If three sensors are situated in one probe, for all the components of EM field, then the output voltage of the sensors will be [4]:

$$
\begin{aligned}
& U_{x 1}=U_{x}+Z_{x y} I_{y}+Z_{x z} I_{z} \\
& U_{y 1}=U_{y}+Z_{y x} I_{x}+Z_{y z} I_{z} \\
& U_{z 1}=U_{z}+Z_{z x} I_{x}+Z_{z y} I_{y}
\end{aligned}
$$

One can see that the sensors interact mutually, which results in a change of the output voltage. This influence brings to the measurement of EM field a systematic error, which can be expressed [2], e.g., for x-axis sensor, in the presence of another sensor as a percentage:

$$
\delta_{U_{x}}=\frac{Z_{x y} I_{y}}{U_{x}} \cdot 100 \%
$$

and in the presence of the two other sensors:

$$
\delta_{U_{x}}=\frac{Z_{x y} I_{y}+Z_{x z} I_{z}}{U_{x}} \cdot 100 \%
$$

\section{NUMERICAL CALCULATION}

Mutual impedance of the EM field sensors and its impact on the induced voltage at the sensor terminals may be calculated analytically or numerically. However, analytical calculation is very complex and it exceeds the scope of this work. Therefore, we will pay attention rather to numerical calculation, which may be used to calculate the mutual impedance between the sensors as well as the induced voltage at their terminals.

The numerical calculation was carried out using FEKO EM field solver. FEKO is based on the method of moments (MOM), a numerical technique used to convert these integral equations into a linear system that can be solved numerically using a computer [6], [7].

First, the sensor is placed into the planar wave EM field. The sensor is a wire (dipole) oriented to be tangentially to the electric field vector - E (Fig.1.). Next, the dipole's orientation is changed perpendicularly to the $\mathrm{E}$ vector, as Fig.2. shows. The intensity of E-field was $1 \mathrm{~V} / \mathrm{m}$ and frequency was $477 \mathrm{MHz}$ (resonant frequency for all sensors).

In both cases, the sensors were loaded by impedance of $75 \Omega$. In case of Fig.1., the calculated current is $1.35 \mathrm{~mA}$, flowing via mentioned impedance. In case of Fig.2., the current is zero-value. The absolute value of the input impedance of the sensor in Fig.1. was $70.438 \Omega$.

Next, the model will consist of two sensors located very closely to each other, as it is shown in Fig.3. It is obvious from Fig.3. that one sensor is located tangentially and the other normally to the electric vector component of the incident EM field.

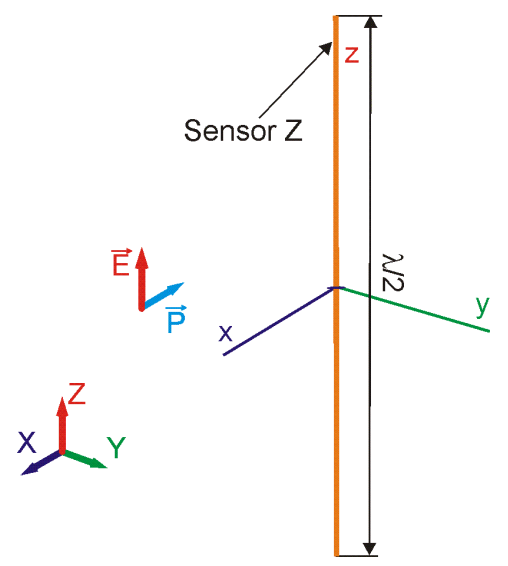

Fig.1. Impact of EM wave on tangentially oriented sensor.

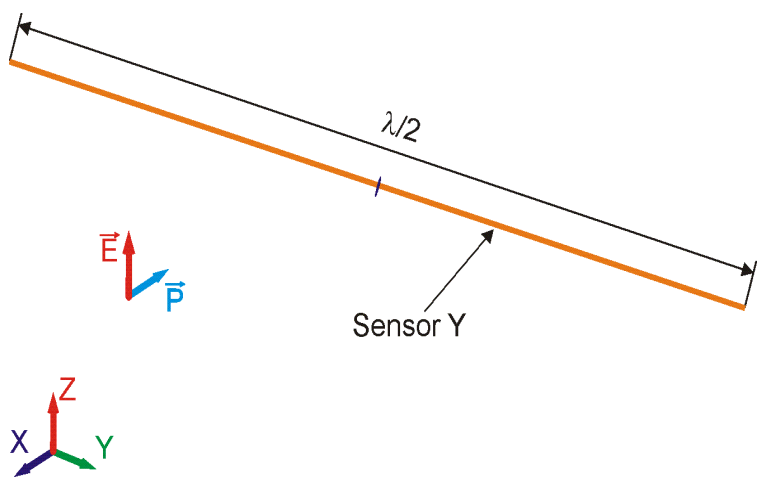

Fig.2. Impact of EM wave on normally oriented sensor.

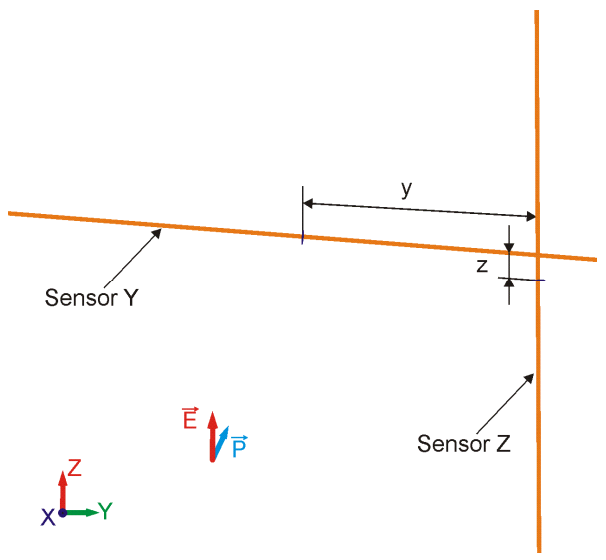

Fig. 3. Arrangement of two sensors in EM field.

\section{RESULTS}

Based on simulation models (Fig.1., Fig.2. and Fig.3.) we performed the calculation of input impedance of the sensor positioned on the z-axis, which depends on the position of the sensor situated on the y-axis. Fig.4. and Fig.5. show only the change of the input impedance of the z-sensor. One can see that the input impedance variation of the examined dipole will be zero if the second dipole is placed in a considerable distance from the examined dipole. A similar effect may be reached by locating the second sensor near the center or at the end of the examined sensor. The design of the EM field probe has to be based on Fig.4. and Fig.5. to minimize the interactions between the sensors. 
Next, we focused on the calculation of the induced current on the y-sensor, which is oriented normally to the E vector of the incident EM field (see Fig.6. and Fig.7.). Unlike the previous case, the induced current takes a zero value only if the examined sensors are sufficiently far apart.

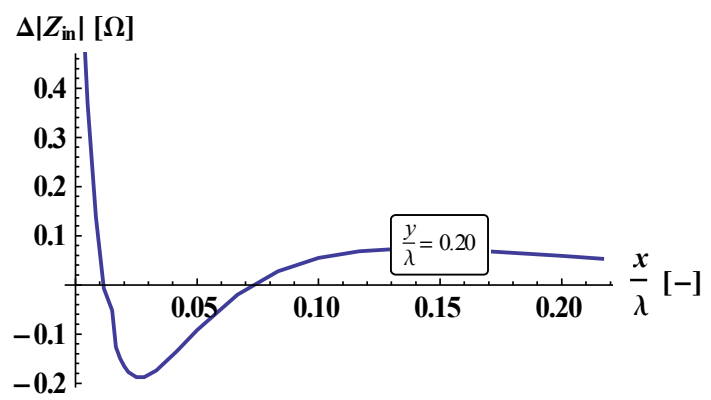

Fig.4. Dependence of input impedance variation in case of $\mathrm{z}$-sensor on relative position of the sensors - shift in $\mathrm{x}$-direction.

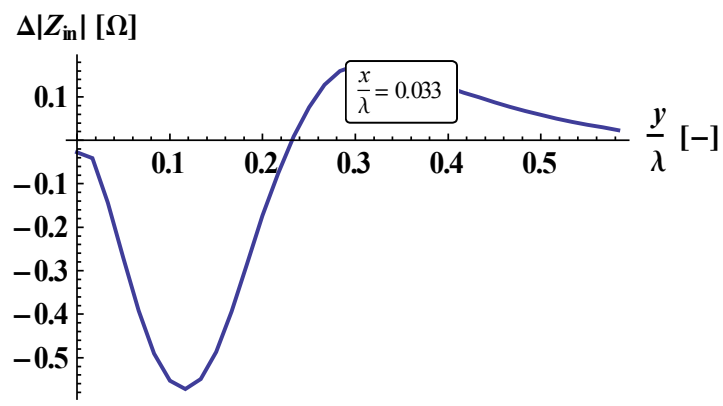

Fig.5. Dependence of input impedance variation in case of $\mathrm{z}$-sensor on relative position of the sensors - shift in y-direction.

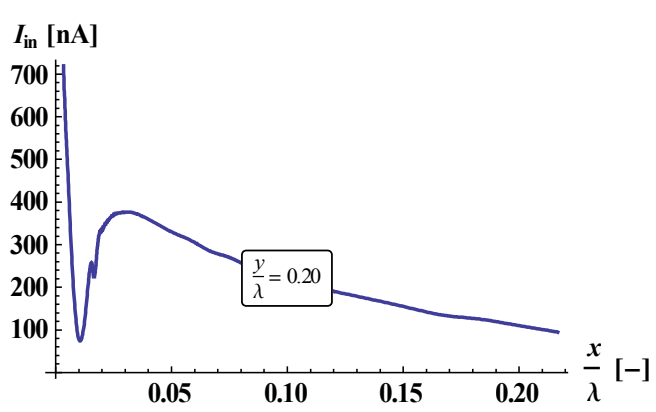

Fig.6. Induced current of y-sensor depending on relative position of the sensors - shift in x-direction.

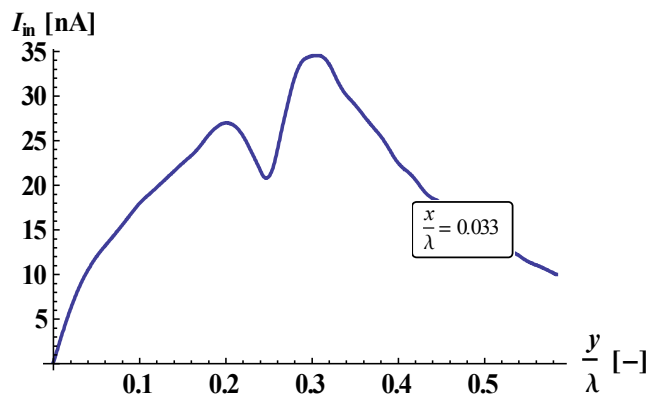

Fig.7. Induced current of y-sensor depending on relative position of the sensors - shift in y-direction.
To quantify the effect of mutual impedance on the accuracy of the EM field measurement, we have to build on Fig.4. to Fig.7., and the modified equation (1) valid for two sensors:

$$
\begin{aligned}
& \delta U_{E}=\frac{\sqrt{\left(Z_{i n Z} \cdot I_{i n Z}\right)^{2}+\left(Z_{i n Y} \cdot I_{i n Y}\right)^{2}}-Z_{0 Z} \cdot I_{i n Z}}{Z_{0 Z} \cdot I_{i n Z}} 100 \% \\
& =\frac{\sqrt{\left(Z_{i n Z} \cdot 1.35 m A\right)^{2}+\left(75 \cdot I_{i n Y}\right)^{2}}-70.43 \cdot 1.35 m A}{70.43 \cdot 1.35 m A} 100 \%
\end{aligned}
$$

Shape of the relative error caused by the existence of the mutual impedance is the same as it is the case shown in Fig.4. and Fig.5. In case of another sensor located on the yaxis, the specific values of E-field measurement relative error on z-axis are shown in Fig.8. and Fig.9.

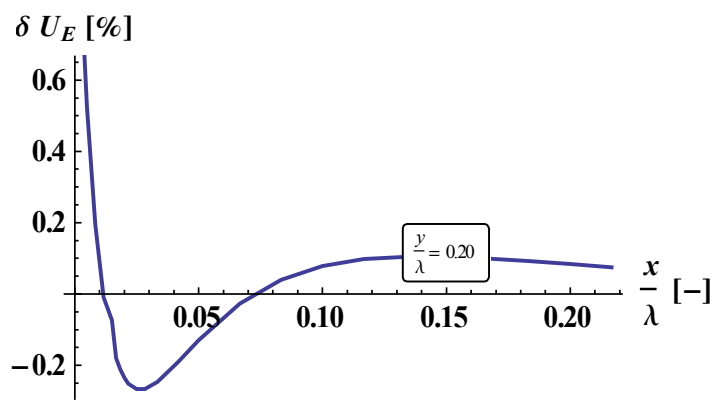

Fig.8. Relative error of $z$-sensor induced voltage depending on relative position of the sensors - shift in $\mathrm{x}$-direction.

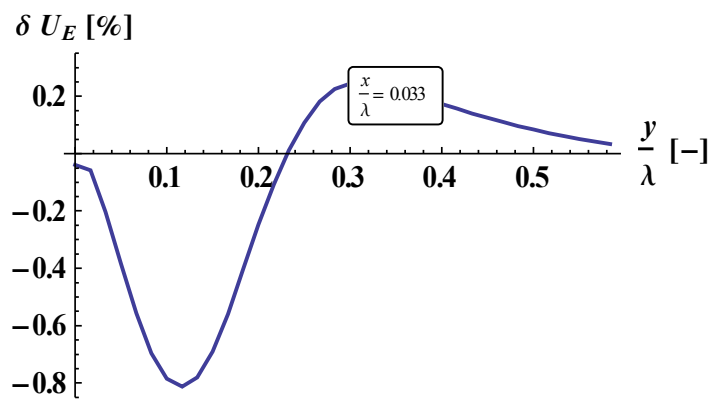

Fig.9. Relative error of $z$-sensor induced voltage depending on relative position of the sensors - shift in y-direction.

As we can see, the relative error of sensor induced voltage, if the y-sensor is involved, does not exceed $1 \%$ value of any considered combination of mutual positions. Thus, we can conclude that the mutual impedance does not have any significant influence on the EM field measurement error.

\section{CONCLUSION}

In case of the EM field measurement with multiple sensors, which are situated close to each other, there is the mutual impedance between the sensors, which may affect the measured results by up to $1 \%$ error, depending on relative positions of the sensors. 
The influence of phantom voltage on EM field measurement error was not analyzed in this paper. This kind of voltage is coming from (2) basis and is also discussed in [3] and [5]. The reason of that is the interaction of EM field and the corresponding sensors. This topic will be taken into account in our future work.

\section{ACKNOWLEDGMENT}

This work was supported by the Slovak Research and Development Agency under the contract No. APVV-033311 and by the project VEGA 1/0963/12.

\section{REFERENCES}

[1] Hajach, P., Hart'anský, R. (2000). Solution of resistive loaded dipoles by Hallen method and by moment method. In Radioelektronika 2000 : 10th International Scientific Conference, 12-13 September 2000. Bratislava: FEI STU, IV62-IV64.

[2] Hart'anský, R., Bittera, M. (2001) Assurance of nondirective pattern of electromagnetic field sensor. In 4 th International Symposium on Mechatronics, June 2001. Trenčín: TnUAD, 164-168.
[3] Hart’anský, R., Slížik, J., Maršálka, L. (2013) Dipole near field analysis - a closed form calculation in cartesian coordinates. Journal of Electrical Engineering, 64 (5), 327-330.

[4] Balanis, C.A. (1997) Antenna Theory: Analysis and Design, 2nd edition. John Wiley \& Sons.

[5] Slížik, J., Hart’anský, R. (2013) Metrology of electromagnetic intensity measurement in near field. Quality Innovation Prosperity, 17 (1), 57-66.

[6] Bittera, M., Kováč, K., Hallon, J. (2007) Measurement of semi-anechoic chamber using modified VSWR method above 1GHz. Measurement Science Review, 7 (3), 42-46.

[7] Bittera, M., Smieško, V., Kováč, K., Hallon, J. (2010) Directional properties of the bilog antenna as a source of radiated electromagnetic interference measurement uncertainty. IET Microwaves Antennas \& Propagation, 4 (10), 1469-1474.

[8] Walton, C.G. (2008) The Method of Moments in Electromagnetics. Chapman \& Hall/CRC.

Received July 15, 2013. Accepted November 29, 2013. 